\title{
Dose Dependent Effects of Retinoids on the Heart of Swiss Albino Mice
}

\author{
Dr Rashmi Sharma and Ashok Gupta \\ Department of Zoology SPC GCA AJMER M.D.S.U. Ajmer Rajasthan INDIA
}

\begin{abstract}
The specific branch of developmental biology which deals with abnormalities caused as a result of failure of normal pattern of development is referred as teratogenesis. Retinoids are essential for spermatogenesis, oogenesis, placental development, foetal morphogenesis and growth. The role of vitamin A in vision is well known. Active derivative of vitamin A (Retinoids) play an important and multiple role in Mammalian development and homeostasis (Sapin, et.al. 1997, Dup et al 1997). These essential dietary compounds are needed in very small quantities, they are not synthesized by the animals and are obtained from external sources in the form of $\beta$ carotene (C40H56). In cells of intestine $\beta$ carotene is converted into vitamin $A$ alcohol and is transported by blood to liver where it is esterfied and stored as vitamin A palmitate. RA is not stored in liver but is derived from retinal and it is biologically the most potent form of vitamin A. Vitamins are organic compounds which are devided into 2 categories $i$ Fat soluble $(A, D, E, K)$ and ii water soluble $(B$ and $C)$. Vitamin A belongs to fat soluble category of vitamins. A fat soluble substance essential for life was first discovered in egg yolk in 1909 by Stepp (Pawson, 1981; Robert and Sporn, 1984). It was confirmed by Mc Collum Devis in 1913 when he found a compound with similar biological activity in butter fats, egg yolk and cod liver oil and was named fat soluble vitamin A. The development of an organism is a complex process of embryogenesis involving cell proliferation, differentiation, migration and organogenesis. Many agents interfering the development process can cause malformations in the embryo. The study of these congenital abnormalities is called teratology and agents which are responsible for causing these malformations are called teratogens. Susceptibility to teratogens depends on the genotype of the organism, including species as well as strain differences. Etienne Geoffrey Saint Hilaire and his son in 1820 started experiments on chick embryo by disturbing its environment in different ways at different embryonic stages. They found some anomalies like Trioncephally, atrophy of eyes and spina bifida in his experiments (Tuli, 1968). In 1877 Dareste reported found some anomalies like Trioncephally, atrophy of eyes and spina bifida in his Experiments (Tuli, 1968). RA severely damaged skin on later stage ie 11th and 14th day gestation period, of development, shows disruption and folds of skin look like over varying skin
\end{abstract}

Keywords: Retinoids, Teratology, Swiss albino mice, Liver.

\section{Introduction}

The branch of development biology which deals with abnormalities of development or failure of normal development is referred as teratology. Retinoids are essential for spermatogenesis oogenesis, placental development, foetal morphogenesis and growth. The role of vitamin A in vision is well known . Active derivative of vitamin A (Retinoids ) play an important and multiple role in Mammalian development and homeostasis (Sapin, et.al. 1997, Dup et al 1997). These essential dietary compounds are needed in very small quantities, they are not synthesized by the animals and are obtained from external sources in the form of $\beta$ carotene $(\mathrm{C} 40 \mathrm{H} 56)$. In cells of intestine $\beta$ carotene is converted into vitamin $\mathrm{A}$ alcohol and is transported by blood to liver where it is esterfied and stored as vitamin A palmitate. RA is not stored in liver but is derived from retinal and it is biologically the most potent form of vitamin A.Vitamin A belongs to fat soluble category of vitamins. A fat soluble substance essential for life was first discovered in egg yolk in 1909 by Stepp (Pawson, 1981; Robert and Sporn, 1984). It was confirmed by Mc Collum Devis in 1913 when he found a compound with similar biological activity in butter fats, egg yolk and cod liver oil and was named fat soluble vitamin A. The name of vitamin was given by Dummond In 1920. The natural and synthetically obtained vitamin A is found in several forms of alcohol (Retinol), aldehyde ( Retinol palmitate ), acetate and retinoic acid. They were collectively known as retinoids. Retinol or alcoholic form of vitamin A is a unstable organic compound. They are easily oxidized specially in the presence of heat. The ester form of vitamin A are fairly stable. When vitamin A is taken in the form of esters (Palmitate/acetate), its converted into alcoholic form (Retinol) in the intestinal cells to be carried to liver, where it is converted to palmitate form for storage. Retinoic acid is not stored in the liver but it is derived from retinol and it is biologically most potent form. The development of an organism is a complex process of embryogenesis involving cell proliferation, differentiation, migration and organogenesis. Many agents interfering the development process can cause malformations in the embryo. The study of these congenital abnormalities is called teratology and agents which are responsible for causing these malformations 
are called teratogens. Susceptibility to teratogens depends on the genotype of the organism, including species as well as strain differences. Teratological experiments was started from 1820s with the studies of Etienne Geoffrey Saint Hilaire and his son on chick embryo by disturbing its environment in different ways at different embryonic stages. They found some anomalies like Trioncephally, atrophy of eyes and spina bifida in his Experiments (Tuli, 1968). In 1877 Dareste reported some congenital malformation in the chick embryo by environmental disturbance.

\section{Materials and Methods}

The mice were obtained from mice breeding center, Department of Zoology M.D.S.U. Ajmer. Animals were fed synthetic diet mice feed pallets (Brook Bond Lipton India Ltd.) Supplemented with germinated grains, seasonal green vegetables, multivitamin drops and water ad libitum. Tetracycline mixed in water was regularly given. There are four virgin female mice $25+-1 \mathrm{gm}$ caged with fertile healthy male in the evening and these were examined for presence of vaginal plug, the next morning. The day appearance of vaginal plug was considered as day 0 of pregnancy.

\section{The veginal plug}

After copulation a secretion of seminal vesicles in the ejaculate of the male coagulate to form a plug in the vegina extending from the cervix to the valve, where it is ordinarilyvisible and is a convenient external sign that mating has occurred (Green, 1996).

\section{Duration of pregnancy}

The gestation period in this species is 19 days and occasionally 20 days. The youngs are most frequently born in the early hours of the morning between midnight and 4 AM.

\section{Objectives}

Investigation of the effects of retinoic acid on different developmental stages (5th, 8th, 11th,and 14th) of mouse embryo.To study effects of RA on organogenesis of skin, liver, heart .Effect of RA on skeletal elements.

\section{Experimental design}

The pregnant females were devided into following groups :(6 animals per group ) Group A : untreated Group B : Treated

\section{Doses}

Suitable (non lethal or sublethal) doses of RA were screened on mice embryos on different stages of developments. The following doses were found to produce various teratological defects on developing mice embryos

1. 2mg RA /pregnant female.

2. $4 \mathrm{mg}$ RA /pregnant female.

\section{Effects of RA on heart}

\section{Results and Discussions}

The cardiovascular system is the portion of the circulatory system that includes heart and blood vessels. It moves the blood between the body cells and the organ of integumentary, digestive , respiratory and urinary system that communicate with the external environment. In performing this function the heart act as pump that forces blood through the blood vessels. The blood vessels in turn form a close system of ducts which transport the blood and allows exchange of gasses, nutrients and wastes between blood and body cells. The circulatory system is one of the great achievement of the lateral plate mesoderm consisting of heart, blood cell and an intricate system of blood vessels. The circulatory system provide nourishment to the developing vertebrate embryo. The circulatory system is 1st functional unit in the developing embryo and the heart is the first functional organ. The heart is hollow cone shaped muscular pump located within the mediastinum of the thorax and resting upon the diaphragm. In vertebrate embryos, the heart develops in an anterior position, within the splanchnic mesoderm of the neck and later moved to the chest. In amphibians, the two presumptive heart forming regions are initially found at the most anterior position of the mesodermal mantle, while the embryo is going neurulation, these two regions come together in the ventral region of the embryo to form a common pericardial cavity. In birds and mammals heart also develops by fusion of paired primordial, but the fusion of these two rudiments does not occur until much later dev. In amniote the embryo in a flattened disc and the lateral plate mesoderm does not completely encirc le the yolk. The presumptive heart cells migrate to the lateral side of the mesoderm ( at the level of hensens node ) when the embryo is 6-7 hours old. At this time embryo is 
still simple consisting of 3 germ layers as the presumptive heart cells move between ectoderm and endoderm towards the middle of the embryo they remain in close contact with the endoderm.(Linsak and lash 1986). The presumptive heart cells of bird and mammals form a double walled tube consisting of inner endoderm and outer epimyocardium. The endocardium will form the inner lining of the heart muscles which will pump for life time of organism. The walls of heart is composed of three distinct layers outer epicardium, middle myocardium and inner endocardium. Epicardium corresponds to the visceral pericardium, functions as outer protective layer. It consist of connective tissues covered by epitheliam and includes blood capillaries, lymph capillaries and nerve fibers. The deeper portion of epicardium contains fat, along the path of larger blood vessels. The mid layer of myocardium is relatively thick and consist largely of the cardiac muscle tissues responsible for forcing the blood out of the heart chambers. The muscle fibers are arranged in planes separated by connective tissues that are richly supplied with blood capillaries, lymph capillarie The endocardium lines all heart chambers and covers the structure such as heart valve. The inner lining is also continuos with inner lining of blood vessels. (endothelium ) attached to the heart. Internally heart is devided inton 4 chambers 2 left and 2 right . The upper chamber called Atria (atrium) have relatively thin walls and receive blood from veins. small ear like projections called auricles extend outward from atria. The lower chambers the ventricles force blood out of the heart into arteries. The atrium and ventricle on the right side are separated from those of the left by septum. The right atrium receives blood from the 2 large veins the superior vena cava and the inferior vena cava. These veins return blood that is low in oxygen from various body parts. The left atrium receives atrium from lungs through 4 pulmonary veins 2 from right lungs and 2 from left lung. The blood passes from left atrium to left ventricle through atrioventricular valve (AV Valve). The right ventricle has a thinner muscular wall than the left ventricle.The right chamber pumps the blood fairly short distance to the lungs against a relatively low resistance to blood flow. The left ventricle on the other hand must force the blood to all the other part of the body against a much greater resistance to flows. When the muscular wall of the right ventricle contracts , the blood inside the chamber is put under increasing pressure. Left ventricle Receives oxygenated blood from the left atrium and pumps it to the aorta. It forms the apex , a part of the stenocostan surface most of the left border

\section{2mg RA on 5th day gestation}

Treat of pregnant female with RA $2 \mathrm{mg}$ pregnant female on 5th day gestation does not inhibit development of heart. Heart appeared normal in morphogenesis.

\section{2mg RA on 8th day gestation}

Cause slight bulging of right ventricle, the prominent notch is observed between left ventricle and right ventricle. The lower conical portion of the heart known as apex, represented by the left ventricle is also distinct.The CS (cross section ) of the heart show degeneration of epicardium. The organization of myocardium also deformed particularly in the right ventricle. The trabencule which are normally aligned along the endocardium of the heart ,gets radially originated. Many nuclei particularly in the myocardium are condensed and appeared as dark stained dots.Such nuclei are picnotic which ultimately becomes necrotic or dead. The intra ventricular sepum is uniformly thickened in the untreated cases whereas a narrow in dorsal surface of the heart of RA treated cases.

\section{2mg RA on 11th day gestation}

The effect produced on ventricular part of heart. Bulging of right ventricle is observed. Ventricle of RA treated heart reduced in length and became wide. A notch observed between right and left ventricle. The apex represented by left ventricle is also reduced. The CS shows the coronary artery in epicardium became enlarged particularl;y in right ventricle. The pericardium shows detachment at certain points. Nuclei dark strained condensed bobies. Trbenculaes of endocardium aline to the wall of ventricle in the lumen. These are radially organized. The lumen is reduced. Left and right ventricle septum is badly disorganized.

\section{2mg RA on 14th day gestation.}

The shape of hearts is very abnormal. There is enlargement of ventricular region. The ventricles show slight contour both in right and left sides. The ventricle appears wheatgrain in shape. Slight enlargement of right and left ventricle has resulted In abnormal shape in lower portion of the heart. Intraventricuar septum is abnormal in the ventricle part i.e. many radiating fibers are present in this region with septum. The wall of the right ventricle is very thin as compared to left. The trebencule of right ventricle become disorganized.

\section{4mg RA on 8th day gestation}

The higher concentration of RA cause further severity with very enlarged and distinct contours. The notch between left and right ventricle is distinct. Disorganizing the conical shape of ventricular end of the heart. Coronary arteries are widened and more branched. Internal organization shows clear lumen separated by 
intraventricular septum. The endocardium lining are showing radiating trabenculae. Pericardium shows detachment through out the ventricle. Coronary arteries are wide. Nuclei of heart muscles are not healthy and shows condensation.

\section{4mg RA on 11th day gestation}

Shows highest rank of teratological defects in the heart. The morphology of heart appears very abnormal. The conical shape of ventrical disappeared. Right and left ventricles become enlarged. In between right and left ventricle in the epical region a small outgrowth is also seen. Right and left ventricle are also e xtended. CS shows reduced lumen of left and right ventricle. Pericardium shows detachment. Endocardial and myocardial layer get hypertrophized and their muscle fiber are conversing become the lumen of heart. Intraventricular septum hypertrophised. Its lateral wall extending towards lumen. The trabencule are radiating, leaving wide inter trabencular space. Coronary arteries present in epicardium are wide. Nuclei shows condensation indicating picnotic condition.

\section{4mg RA 14th day gestation}

The auricle are extended covering the middle portion of ventricle. These are globular in shape. There is extensive enlargement of right ventricle. This appears another apex in addition to apex by left ventricle. In the lowermost region of heart notch is distinct in between both ventricles . Both ventricles show increased width. CS shows ventricular region become flattened shape (untreated) to rounded structure (treated). It means the normal dorsoventral flattened structure of ventricle becomes globular. The intraventricular septum has extended from its lateral walls. Endocardium fiber radiating towards the lumen. Lumen of both ventricles is reduced and occupied by trabenculaes arrangement in radial fashion and at some places even bundles of these muscles fibers. Intraventricular septum shows long connective tissue fibers. In certain regions large number of pictonic cells are observed in walls of the heart.

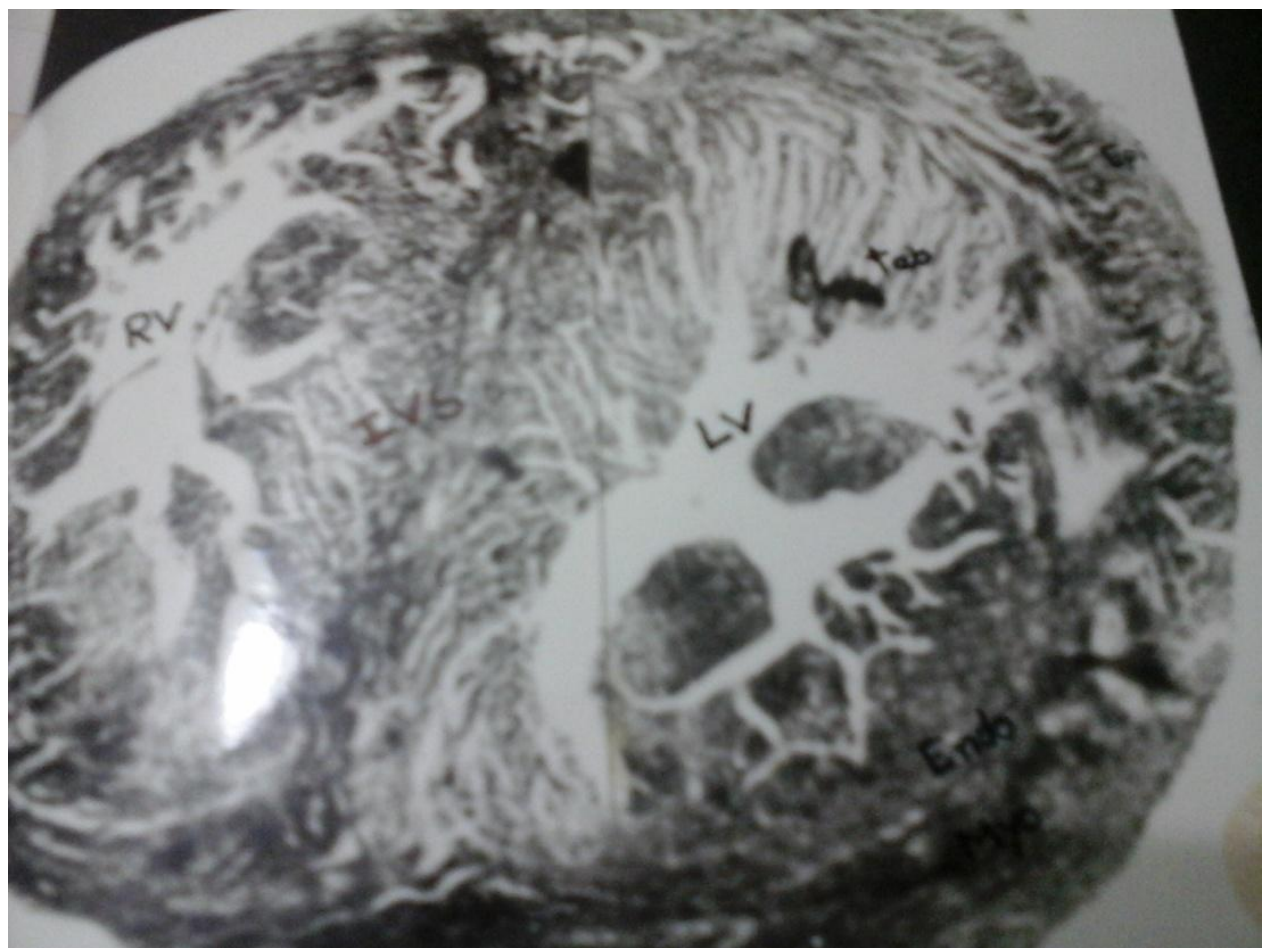

Image 1 Histological picture of heart of newly born mice treated with 4mgRA/pregnant female at 14th day gestation, TS through ventricle region showing slightly reduced lumen.

Pc-Pericardium, Epi- Epicardium, Myo- Myocardium, Endo- Endocardium, IVS- Intraventricular septum, Pf-Purkinje fibre, tab-Trabanculae, CA-Coronary artery,BV-blood vessel,RV-Right ventricle, LV-Left ventricle. 


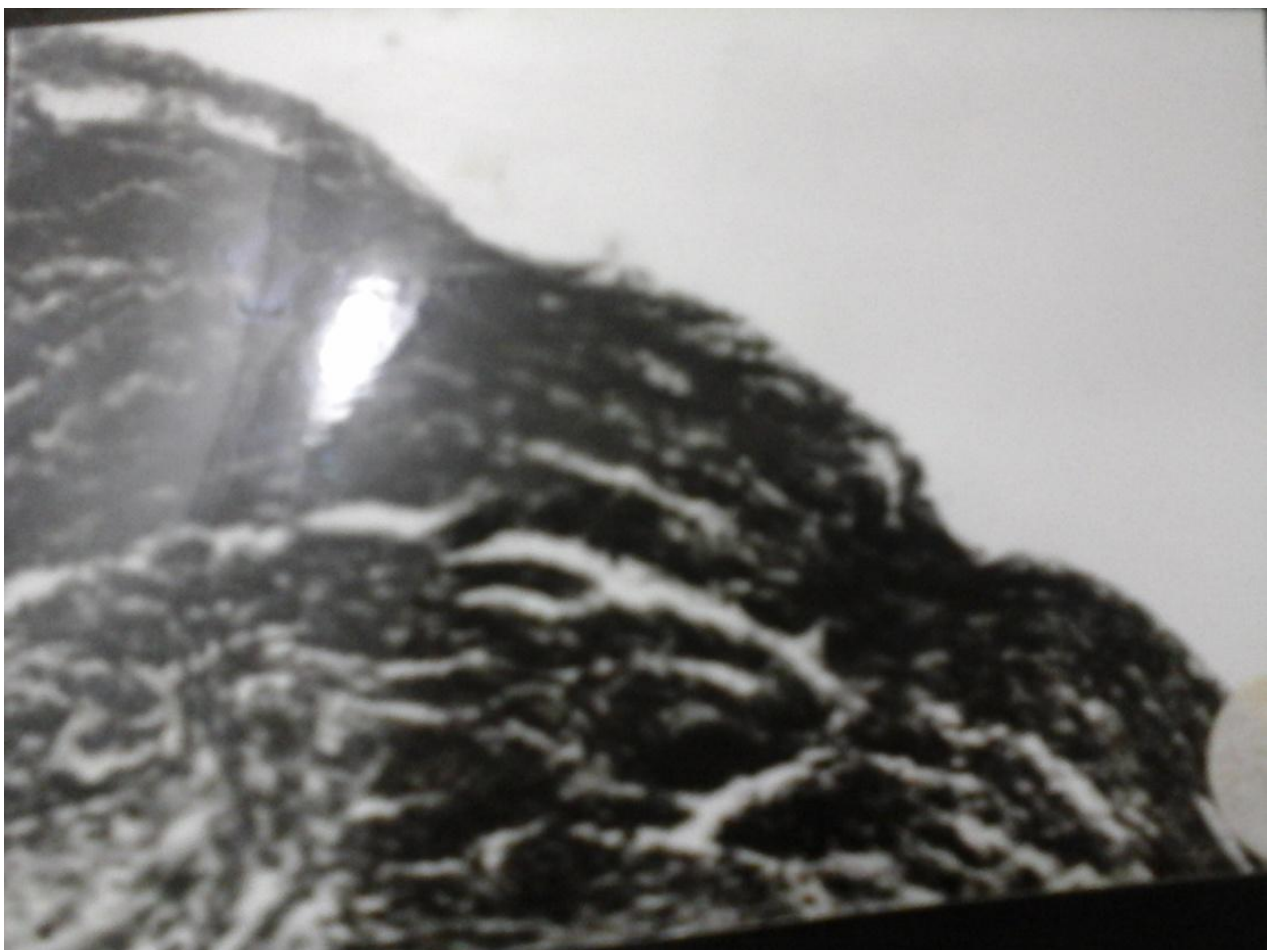

Image 2 Magnified view of layers of right ventriclesas seen in figure.

Pc-Pericardium, Epi- Epicardium, Myo- Myocardium, Endo- Endocardium, IVS- Intraventricular septum, Pf-Purkinje fibre, tab-Trabanculae, CA-Coronary artery,BV-blood vessel,RV-Right ventricle, LVLeft ventricle.



Image 3 Magnified view of cardiac layers of right ventricle with septum as seen in figure showing disrupted myocardial fiber. 
Pc-Pericardium, Epi- Epicardium, Myo- Myocardium, Endo- Endocardium, IVS- Intraventricular septum, Pf-Purkinje fibre, tab-Trabanculae, CA-Coronary artery,BV-blood vessel,RV-Right ventricle, LV-Left ventricle.

\section{Conclusion and Summary}

Development of heart start with the formation of somites and blood islets . Heart rudiments form mesoderm and endoderm on 7 day 21 hours to 8 day 21 hours. The development of heart and cardiovascular system takes place in a coordinated manner and any intervention during this process may disorganize the morphogenesis of heart and cardiovascular system. The development of heart o birds and mammals is dependent on optimum conc. Of RA(Thompson et.al., 1969, dickman, 1997). The optimum conc. of Retinoids are required for a normal development of cardiovascular system but an excess quantity of Retinoid produces variety of abnormalities of cardiovascular system. when 5th, 8th, 11th, 14th, day gestation pregnant females were given 2 or $4 \mathrm{mg}$ oral oral dose of RA the treatment resulted variety of teratogenic effects. The effect were dose and stage dependent . 11th day gestation female treated with $4 \mathrm{mg}$ RA showed enlargement of right ventricle, disorganized musculature of the wall of heart, thickening of heart wall and septum obliterated lumen of heart, enlarged coronary arteries etc. In the case of extreme severity shape of ventricle so badly differentiated that instead of appearing conical shape it appeared $\mathrm{w}$ shaped. Kalter and Warkany, (1961) has also found malformation of heart and vessels. Narrow and angulated ductus arteriosus, complete transposition of great vessels, high intraventricular septal defects and probable coronary arteries occupying a large space between the ventricles Kim et.al. (1995)observed in atrium and ventricular region, intraventricular valve, intraventricular septal defects, pulmonary artery, hypoplasia of aortic arch anomalies. Rosa et.al. (1986)also reported that effect of hypervitaminosis of new born human babies have shown cardiovascular malformation including transportation of great vessels and ventricular septal defects etc. In children suffering from severe hypervitaminosis treatment of RA causes enlargement of heart at early mid (48-72 hr) stage of development (Singh, 1995, Shobhawat 1998). Malformation produced by RA depend on particular type of morphogenesis undergoing in developing embryo of treated females when processing of pericardium formation are going on during the early mid phase (8th-11th dgp) RA treatment cause detachment of pericardium from the outermost layer of heart. Chambers of heart, septum and its wall takes place between 8th to 14th day gestation Treatment of pregnant female during this period cause disorganization of these structures of heart. 5th day process of morphogenesis of heart and heart chambers does not start. So 5th day treatment is not much affected. When 14th day gestation treatment female was observed in the newly born mice morphogenesis was affected much as compared to 5th day gestation in the 14th day gestation treated cases most of the foundation process of morphogenesis are completed. The RA treatment did not influence much the heart morphogenesis at this developing stage. 4mg RA 8th day gestation shows branching of portal vein but not much enlargement in central vein and bile duct. RA disrupt the layer of cardium and blood vessels. Heart is seen as swollen, its lumen is obliterated the characteristic trabancules of Ventricular wall are obliterated. Causes enlargement of right ventricle. RA disorganized 3 fundamental layers of heart namely epicardium ,myocardium and endo- cardium. Reduce lumen of ventricle and in certain cases the intraventricular septum losses the characteristic of septum. Trabenculae become radially organized in endocardium. Detachment of pericardium. Abnormality of coronary artery(number and size of vessels). Enlargement of right atrium in late stage embryo.11th day gestation is more sensitive as compared to 8th and 14th day gestation stage.

\section{References}

[1]. Coblan, S.Q.(1953):Excessive intake of Vit A as a cause of congenital anomalies in Rat. Science 117:535-536.

[2]. Coblan , S.Q. (1954):Congenital anomalies in rat produced by excessive intake of vit A during pregnancy. Pediatrics. 13 556-557.

[3]. Dickman , E.D. and Smith, S.M. (1996) Selective regulation of cardiomyocyte expression and cardiac morphogenesis by RA dev dynamics 206(1):39-48.

[4]. Effendy. I., (1996): Differential irritant skin responses to topical RA and sodium layryl sulphate alone and in crossover design British J. of Dermatology 134: 420-430. (Abstract).

[5]. Elmzar, M.M.A.(1996). Pattern of Retinoid induced teratogenic effect. Possible relationship with relative selectivity for nuclear retinoid receptors RAR-alpha,RAR-beta and RAR gama. Teratology 53(3):158-167.(Abstract).

[6]. Fisher, G.J. and Voiegwwa, J.J. (1996): molecular mechanisms of retinoid actions in skin,FASEB J.10(9).1002-1013.

[7]. Giroud, R. and Martinet,(1954): Fertes du Polasis chezl embryos de rat per hypervitaminose A., Comp.Rend.Soc.Biol.148:17421743.

[8]. Goodman, A.B. (1996) : Congenital anomalis in relative of Schizophrenic probands may indicate a retinoid pathology. Schizophrenia Research 19: 2-3:169-170.(Abstract).

[9]. Griffiths. C. E. M. (1996): Tropical RA changes the epidermal cell surface glycosylation pattern towards that of a mucosa epithelium. British Journal of Dermatology. 134; 431-436.

[10]. Leelaprute. L .; Boonpucknaving. V.; Bharmar apratuati. N., and Weerapradist. W.(1973). Hypervitaminosis A in rat Arch. Sci 85:42-55.

[11]. Kalter, H.C.(1960): The teratogenic effect of hypervitaminosis upon the face and mice of inbred mice. Ana. N.Y. Acad. Sci.85:4255 . 
[12]. Kalter, H.C. and Warkany J. (1961): Experimental production of congenital malformation In strains of inbred mice by maternal treatment with hypervitaminosis. Amer J. path,381-21. Kochhar, D.M. (1967): Teratogenic activity of Retinoic Acid. Acta Pathol Micro. Biol. Scand. 70:398-404.

[13]. Moore, T. (1957): "Vitamin A “Am. Elsevier, New Delhi. Niazi, I.A. : Pescitelli M.J.; and Stocum, D.L., (1985) ; Stage dependent effects of RA on regenerating urodele limbs. 